\title{
Gradient Diffusion Method
}

National Cancer Institute

\section{Source}

National Cancer Institute. Gradient Diffusion Method. NCI Thesaurus. Code C85599.

A method to determine microbial susceptibility to antibiotics in which an agar plate, which has a concentration gradient of the drug within the agar, is streaked with the microorganism of interest. Microbial susceptibility as well as minimum inhibitory concentration of antibiotic is assessed. 